\title{
Correction: The path to « the Golden Age » for the treatment of metastatic renal cell carcinoma
}

\section{Gilles Pagès}

${ }^{1}$ University of Nice Sophia Antipolis, Institute for Research on Cancer and Aging of Nice, CNRS UMR; INSERM U1081, Centre Antoine Lacassagne, France; Centre Scientifique de Monaco, Biomedical Department, 8 Quai Antoine Ier, Monaco, Principality of Monaco

Published: November 06, 2018

Copyright: Pagès et al. This is an open-access article distributed under the terms of the Creative Commons Attribution License 3.0 (CC BY 3.0), which permits unrestricted use, distribution, and reproduction in any medium, provided the original author and source are credited.

This article has been corrected: In the fifth paragraph of the article, the following sentence has been corrected to read: 'Roelants et al further show that VHL negative cells re-expressing VHL are less sensitive to the GDC-0941/Saracatinib combination.'

In accordance with these considerations, new strategies must be developed to chronicize or even to cure metastatic kidney cancers. The paper of Roelants et al proposes an elegant strategy based on the following rationale: i) several tyrosine kinase receptors are involved in exacerbated angiogenesis and growth of kidney cancers; ii) all tyrosine kinase receptors activate the PI3 Kinase and Src kinase. Hence, targeting paramount signaling pathways down-stream of tyrosine kinase receptors should be highly efficient and less submitted to tumor cell adaptation. The authors have focused on two drugs used in clinical trial; the GDC-0941 (PI3 Kinase inhibitor) and Saracatinib (Src Kinase inhibitor). Independently, these two drugs have a modest activity on different in vitro parameters of tumors cells aggressiveness. However, the authors observed a synergistic anti-tumor effect by combining both drugs. As described above, $V H L$ inactivation happens in $>70 \%$ of renal cancers. However, the VHL wild-type tumors are more aggressive [12]. Roelants et al further show that $V H L$ negative cells re-expressing VHL are less sensitive to the GDC-0941/Saracatinib combination. Therefore their strategy might be relevant for highly aggressive tumors.

Original article: Oncotarget. 2018; 9:31564-31565. https://doi.org/10.18632/oncotarget.25832 